Asian J. Med. Biol. Res. 2020, 6 (2), 138-148; doi: 10.3329/ajmbr.v6i2.48044

\author{
Asian Journal of \\ Medical and Biological Research \\ ISSN 2411-4472 (Print) 2412-5571 (Online) \\ www.ebupress.com/journal/ajmbr
}

Review

\title{
Radiation-induced side effects in breast cancer patients and factors affecting them
}

\author{
Afrin Sultana Chowdhury* and Saheda Tamanna \\ Department of Biotechnology and Genetic Engineering, Noakhali Science and Technology University, \\ Noakhali-3814, Bangladesh
}

*Corresponding author: Afrin Sultana Chowdhury, Room no.: 602, Department of Biotechnology and Genetic Engineering, Noakhali Science and Technology University, Noakhali-3814, Bangladesh. Phone: +8801834066794; E-mail: afrin.bge@nstu.edu.bd

Received: 26 April 2020/Accepted: 21 June 2020/ Published: 30 June 2020

\begin{abstract}
Breast cancer is the most frequent malignancy and the leading cause of cancer morbidity and mortality in women worldwide. Radiation therapy (RT) is a widely used approach for its treatment. About $50 \%$ of patients with malignant breast tumors receive radiation therapy and most of them appear to tolerate it, but some experience severe side effects induced by this therapy. This variability of response may be caused by several factors, like age, inflammatory responses, body weight and variation in genes involved in the response to radiation-induced DNA damage. To limit radiotherapy side effects in breast cancer patients it is therefore important to have a good knowledge of these associated factors. This review discussed about the radiotherapyrelated side effects in breast cancer patients and the factors affecting them.
\end{abstract}

Keywords: breast cancer; radiation therapy; tissue damage; cardiovascular disorder; genetic makeup

\section{Background}

Breast cancer is the most commonly occurring cancer incidence among women and a leading factor of cancer death globally. In 2012, 1.67 million cases of breast cancer were diagnosed worldwide and were ranked as the fifth major reason of cancer death (522,000 deaths) (Youlden et al., 2012; Knaul et al., 2009).

Although the frequency of breast cancer has increased by $0.2 \%$ per year between 1997 and 2000, improvements in diagnosis and treatment have resulted in significant survival rates of breast cancer patients (Youlden et al., 2014). Several local therapies for breast cancer had considerably diverse effects on the length of survival and quality of life in breast cancer patients. It has long been received that RT can delay or prevent local or regional recurrence in women with early breast cancer (Roychoudhuri et al., 2004).

Radiotherapy is an effective treatment for breast cancer. About $50 \%$ of malignant breast tumor patients receive radiotherapy and most of them appear to tolerate it, but some experience critical side effects induced by this therapy (Borrego et al., 2015) (Hershman et al., 2006). The major side effects of radiation therapy are edema and thickness in the breast, fatigue and sunburn-like alteration in the treated area. These exchanges to the breast tissue and skin commonly go away in 6 to 12 months, and in some cases, the size of the breast becomes smaller and firmer after radiation therapy (Bovelli et al., 2010). Sebaceous glands and hair follicles are more sensitive to relatively low concentrations of radiation and result in the acute side effects of hair loss and skin dryness (Mathes and Alexander, 1996).

Different factors may be responsible for generating this variability, for example, age, inflammatory responses, body weight and variation in genes involved in the response to radiation-induced DNA damage (Lin et al., 2012). Besides, increased exposure to radiation sources and other treatment-related effects may also induce radiation associated with adverse events (Doody et al., 2000). This review is intended to summarize studies concerning radiotherapy-related side effects in breast cancer patients and the factors affecting them. 


\section{Radiotherapy}

Radiotherapy (RT) is a widely used treatment in cancer. The aim of exposing the tumor to radiation is to shrink the tumor mass or to eliminate the tumor cells that may have escaped surgery. The radiation dose is calculated in gray (Gy) units, which is the amount of radiation absorbed in $1 \mathrm{~kg}$ tissue (Dunne et al., 1999). Radiotherapy can be delivered as external beam radiation or as internal radiation. Most of the patients usually receive external radiotherapy and this review will mainly focus on that.

\subsection{Mechanism of radiotherapy}

The major goal of RT is to deprive cancer cells of their multiplication potentially and eventually destroy the cancer cells, while minimizing exposure to normal healthy cells in the body (Lliakis, 1991). RT uses ionizing radiation for more than a century to treat cancer which is mainly based on the rationality that the rapidly proliferating cancer cells are more sensitive to radiation therapy than the normal healthy cells (Schaue and McBride, 2015).

The biological effects of RT can be resulted by the direct or indirect function of radiation on the DNA molecules. In the direct action, ionizing radiation hits the targeted DNA molecule directly, and produce DNA breaks, specifically, Double Strand Breaks (DSBs) (Mladenov et al., 2013). DSBs are difficult to repair and can lead to dramatic chromosomal abnormalities and genetic deletions. Therefore, DSBs increases the probability that cells will undergo cell death (Bassing and Alt, 2004). Previous studies indicated that radiation therapy like the most anticancer treatments achieve its therapeutic effect through inducing DNA damage and therefore cell death (Baskar et al., 2012). Several studies also found that the DNA of cancer cells repair more slowly and also develop more DNA breaks than the normal cells (Halazonetis et al., 2008).

Ionizing radiation can also induce indirect effects to damage DNA by generating reactive oxygen species (ROS) from water molecules of the cell (Bandyopadhyay et al., 1999). ROS are free radicals and mainly categorized by an unpaired electron in the structure. Therefore, they are highly reactive and can react with DNA molecules to result in molecular structural damage (Yang et al., 2013). ROS oxidize proteins and lipids, and induce damages to DNA, for example, generation of single-strand breaks (SSB) and apurinic/ apyrimidinic sites (abasic sites). All these changes jointly induce cell death and mitotic failure (Aparicio et al., 2014) (Redon et al., 2010).

For ionizing radiations including gamma-rays, LET X-rays, $60 \%$ of cellular damage is caused by the indirect effects (Hill, 2004). Both direct and indirect damage to DNA in the form of DNA breakage or replication stress collectively result in a complex DNA damage response (DDR). DDRs contain events that coordinate DNA repair, control of DNA replication, chromatin remodeling and apoptosis (Hershman et al., 2006). The ultimate result of the direct and indirect effects of RT is the biological and physiological changes that may visible in seconds or decades later.

\section{Box 1 | Normal Tissue Tolerance}

The degree of structural damage to a tissue usually depends on the cell's radio-sensitivity. The amount of ionizing radiation required to destroy the functional capability of a matured differentiated cell is much higher than that for dividing cells. The response of a tissue or organ to RT mainly depends on the cell's inherited sensitivity and also on the organization of cells in a tissue. In breast tissues, severe or grade 3 tissue damage of up to 5\% is acceptable 9 (Dunne, 1999) which also accepts relatively high treatment doses. Therefore, an increased amount of toxicity of radiotherapy in case of breast cancer is acceptable to enhance the probability of cure.

\section{Early side effects of radiotherapy}

The early (acute) radiation reaction is a functional and morphological disorder that occurs in cells and intercellular spaces of tissues during and/or immediately after radiotherapy (Yagoda et al., 2009; Donnelly et al., 2010) (Figure 1). Acute radiation toxicity is most remarkable in tissues that renew fast, and this condition is related to the reduction of functional cells, which are removed as a part of normal tissue turnover and not replaced by damaged stem cells.

Early skin associated side effects due to RT might be considered as a sign of sensitivity toward clinical radiation. In a study with 108 patients who were treated with RT after breast surgery, the most frequent early complications identified were erythema (91.7\%), moist desquamation (35.2\%) and dry desquamation (29.6\%). The acute side effects such as erythema and desquamation resolve rapidly without treatment (Beral et al., 2004). Apart from these, fatigue, lymphoedema and changes in breast color and shape are some common early symptoms after radiation therapy. RT induced early fatigue is common in up to $80 \%$ of breast cancer patients (Mohan et al., 2019). This early fatigue is accompanied by loss of appetite, nausea, vomiting, and of acute 
radiation illness (Hickok et al., 2005). Lymphoedema is the swelling of the hand, arm or breast/chest area caused by a build-up of lymph fluid in the exterior tissues of the body. It can occur due to radiation-induced damage to the lymph nodes under the arm (axilla) and the surrounding area. RT after breast-conserving surgery may cause changes to the breast tissue on the treated side. So the breast may look smaller and different than before or may feel firmer (Donnelly et al., 2010).

\section{Late side effects}

Late side effects progress with time, become more severe, and generally cannot stop or reverse (Newman et al., 1998). The length of the latency period as hard to predict also creates a major complication for the management of patients. Moreover, the appearance of severe late radiation reactions is associated with a risk of serious complications and could permanently reduce the patient's quality of life (Bentzen, 2006) (Figure 1).

\subsection{Tissue damage}

Late radiation reactions, such as fibrosis, necrosis and slowly healing wounds, initiate to appear a few months after exposure and mainly reflect damage to proliferating cell fractions that are crucial for the regeneration of injured tissues (Johansson et al., 2000). Cells that are the progeny of exposed cells may divide and express delayed gene mutations and will bear chromosomal aberrations. This phenomenon is called radiation-induced genomic instability. It can be exposed through delayed lethal mutations (Powell et al., 2002) that may lead to prolonged tissue perturbation within the radiation field (Brown, 1983). According to previous in vitro analysis, radiation-induced genomic instability is likely to be the basis of the phenomenon of delayed lethality (delayed reproductive death, DRD) of cells (Mazurik and Mikhailov, 2004). Ionizing radiation-induced genomic instability transmitted via several generations after radiation therapy through the progeny of surviving cells (Little, 1998). Earlier studies reported that induction of delayed reproductive death or lethal mutation in many mammalian cell systems up to six generations after exposure to ionizing radiation (Suzuki et al., 2003). Previous in vivo analysis reported that radiation-induced genomic instability in hemopoietic stem cells in mouse and man occurred through ionizing radiation which could potentially contribute to leukemogenesis (Finnon $e t$ al., 2012).

\subsection{Inflammation}

Inflammation is considered as a normal biological response that is initiated after cell injury due to infection or cell damage. Growing evidence has shown that RT can modulate the immune system through the upregulation of inflammatory mediators (Di-Maggio et al., 2015). Particularly, the ionizing radiation-related activation of cytokine cascades is vital (Schaue et al., 2012). This process is called damage-induced inflammation (Candeies and Testard, 2015). Cytokine-mediated multicellular interactions initiate the fibrogenic process and vascular injury (Weintraub et al., 2010) which is a long-term effect of radiotherapy. Moreover, in addition to its cytotoxic activity, ionizing radiation can also trigger pro-inflammatory mechanisms in tumor and normal cells that receive sub-lethal doses, through the activation of $\mathrm{NF-KB}$ transcription factor. This links with carcinogenesis, inflammation and radiotherapy resistance (Schaue et al., 2012).

Recent experiments suggest that after radiation exposure removal of apoptotic cells enhances phagocytic cell activity and persistent macrophage activation and neutrophil infiltration. These phenomena continue even after the clearance of apoptotic bodies and may be significant determinants of the long term consequences of radiation exposure (Yu, 2012) (Chen et al., 2002).

\subsection{Risk of second cancer}

There are many pieces of evidence for the association between radiation exposure and carcinogenesis, especially from the epidemiological study of the survivors of atomic bomb irradiation (Preston et al., 2003; Land et al., 2003). It has been indicated that irradiation of surrounding tissues during breast RT can induce the chance of second cancers within these tissues (Harvey and Brinton, 1985; Neugut et al., 1999). From different studies radiation therapy for breast cancer is associated with the risk of developing leukemia, sarcoma, lung cancer, and esophageal cancer (Ahsan and Neugut, 1998). Generally, the latency period between exposure to radiation and the appearance of a second cancer is 10 or more years. Boice et al. (1987) have suggested that cancers resulting from radiation would develop after 10 years for solid tumors and within 5 years for leukemia (Zablotska et al., 2005).

Roychoudhuri et al. (2004) also found similar findings in their study. According to their observation, the risk of developing lung cancer was shown to be significantly increased in the RT cohort compared with the non-RT cohort at both 10-14 and 15 or more years after diagnosis of breast cancer. The remarkable risk of developing 
myeloid leukemia was also monitored at $1-5$ years. The risk of esophageal cancers has found significantly elevated at 15 or more years after diagnosis and RT (Roychoudhuri et al., 2004).

\subsection{Cardiovascular disorders}

Prospective studies report that after RT 50\%-63\% of women have experienced cardiac perfusion defects. Radiologic evidence of irreversible lung fibrosis and associated pulmonary disorders was also reported in 6 to 24 months after RT (Marks et al., 2000; Marks et al., 2005). It is here important to mention that an unhealthy lifestyle and presence of risk factors may also contribute to an elevated risk of cardiovascular disorders (CVD) in irradiated breast cancer patients. A report by Hooning et al. (2007) has described that $32 \%, 26 \%, 10 \%$, and $9 \%$ of women treated with radiotherapy were smokers, had hypertension, hypercholesterolemia, and diabetes mellitus, respectively (Hooning et al., 2007).

Studies have shown that RT may enhance the risk of cardiovascular disease many years after initial breast cancer treatment (Batar et al., 2016). By observing a meta-analysis of eight randomized trials that mainly included approximately 8000 women revealed a $62 \%$ enlarged threat of cardiac death rates in women who received RT. These high rates of CVD deaths occur mainly due to high-volume irradiation to the heart that was commonly utilized in earlier RT protocols (Garcia et al., 2016).

Radiation-induced CVD might occur due to a mixture of both microvascular and macrovascular effects. During the microvascular level, radiation therapy leads to a decrease in capillary density which mainly declines the degree of potential collateral flow and these changes are largely subclinical. This radiation-induced altered capillary density has resulted in both rats (Zagar et al., 2016) and mice after larger ( $\geq 8 \mathrm{~Gy}$ ) local heart doses. This damage to the microvascular network appears to be progressive, depending on the time and dose, suggesting a greater role in the underlying cause of ischemic injury (Wang et al., 2007).

On a macrovascular level, RT accelerates atherosclerosis of larger blood vessels and this result can take years, or even decades to become clinically significant (Lipshultz et al., 2013). During the 1950s and 1960s, it was investigated that cardiac damage has resulted in radiation doses $>40 \mathrm{~Gy}$, whereas lower concentrations were considered to be safe (Guo et al., 2011). Newer RT protocols with lower radiation doses and highly focused radiation beams allow tumors to be targeted more preciously and shield the heart and other healthy tissue from the direct effect of radiation (Adams et al., 2003).

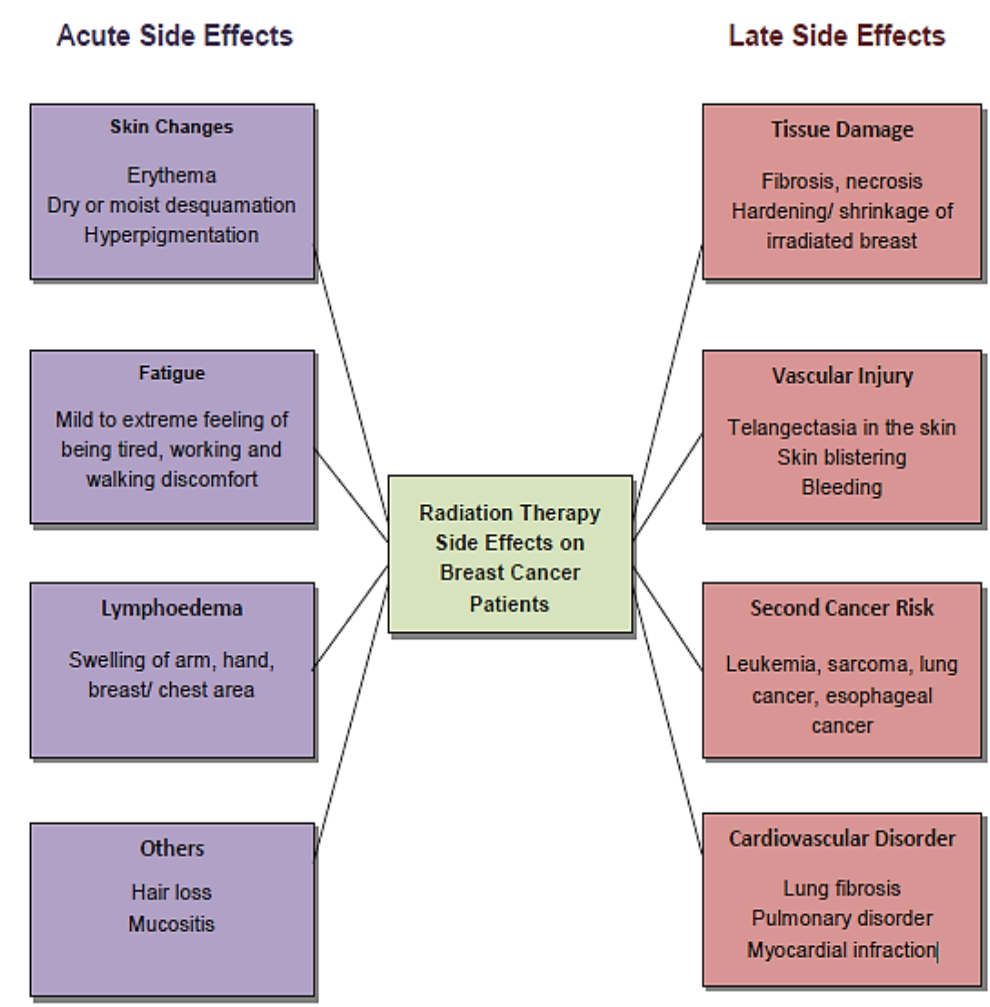

Figure 1. Acute and chronic side effects of radiation therapy on breast cancer patients. 


\section{Factors affecting side effects}

The severity of damage to normal tissue after therapeutic RT is predominantly influenced by factors related to radiation exposure, which however are not sufficient to explain fully due to patient-to-patient variability (Turesson et al., 1996; Rosen et al., 1999). To date more than 60 publications have been published where researchers tried to identify common risk factors for RT side effects. There is significant evidence that both patient- and treatment-associated factors, as well as essential factors of individual radio sensitivity could manipulate the variability of side effects observed (Brock and Tucker, 2000) (Figure 2).

\subsection{Genetic makeup}

Diverse single nucleotide polymorphisms (SNPs) have been proposed to be correlated with acute or late radiation sensitivity. Genome-wide analyses showed that ionizing radiation-related SNPs are not localized in random genes but genes involved in selected processes, such as DNA damage repair, control of apoptosis, cell cycling and inflammation (Brock and Tucker, 2000; Alsner et al., 2008; Popanda et al., 2009; Rosenstein, 2011; West and Barnett, 2011).

The BRCA1 and BRCA2 genes were first identified and sequenced in 1994 and 1995, respectively, after analysis of high risk for breast cancer (Anglian Breast Cancer Study Group, 2000). These genes act as classic tumor suppressor genes in that only one defective copy in the germline results in cancer susceptibility but both copies are lacking in malignant cells. These genes encode large proteins, with the BRCA1 protein-containing of 1863 amino acids and the BRCA2 protein consisting of 3418 amino acids. The exact functions of these proteins are unclear, but they seem to be intimately involved in DNA repair, cell cycle control, recombination and the maintenance of genomic stability (Antoniou et al., 2003). According to previous studies, high complication rates occurred in women with BRCA1/2-associated breast cancers treated with RT (Pierce and Haffty, 2011; Smith and Isaacs, 2007). A retrospective cohort study from Pierce et al. (2011) reported that radiation-associated complications in 71 North American women with a BRCAl/2 mutation with initiative stage breast cancer treated with RT. With a median follow-up of 6.75 years for $B R C A 1 / 2$ carriers and 7.75 years for controls, toxicities were comparable between these groups. The rate of breast pain was enhanced in $B R C A 1 / 2$ carriers. However, other measures of major acute toxicity (breast erythema, moist desquamation, and fatigue) were not significantly different. Late effects (rib fractures, lung fibrosis, soft-tissue/ bone necrosis, and cardiac fibrosis) were also not significantly different between carriers and controls (Pierce and Haffty, 2011).

CDKN1A (cyclin-dependent kinase inhibitor-1A) encodes p21 protein and highly active in cell cycle regulation and arrest following DNA damage and plays a crucial role in breast cancer development (Motwani and Strom, 2006). A recent study of Price et al. (2015) suggested that CDKN1A abnormal expression has been reported to be associated with the acute sensitivity to radiation (Price et al., 2015). Alsbeih et al. (2003) also have shown that individual response in CDKN1A is related to inherent radio-sensitivity (Alsbeih et al., 2003). In another study, researchers have reported an interaction between DNA damage response-related CDKNIA gene expression and the risk of radiotherapy-induced acute side effects (Borrego et al., 2015). Finnon et al. (2012) showed that reduced $C D K N 1 A$ mRNA expression was associated with enhanced normal tissue radiation toxicity by comparing mild and severe acute side effects after 2 Gy irradiation of lymphocyte cultures of breast cancer patients (Finnon et al., 2012).

Earlier studies also resulted that PARPI and $X R C C 1$ gene expression rates were remarkably higher in control than experimental breast cancer patients. Later studies indicated that XRCC1 protein expression levels were significantly enhanced in control vs. experimental cases and higher expression of PARP1 is correlated with large tumor size (Batar et al., 2016). Recent studies showed that abnormal XRCC1 expression levels might be associated with the risk of radiation-induced acute side effects in breast cancer patients (Sasco et al., 2003). Rojo et al. (2012) also suggested that the over-expression of PARP1 protein was highly associated with larger tumor grade and estrogen- negative tumors formation (Rojo et al., 2012).

Cytokines such as transforming growth factor-beta (TGFB1), tumor necrosis factor-alpha (TNF), interferons and interleukins are also involved in the development of radiation induced-toxicity. For example, IL17 receptor knockout mice have enhanced normal tissue radiation toxicity following irradiation (Baum et al., 2005). Recently, a mouse model has reported substantial evidence for the role of TGFB1 in the pathogenesis of breast cancer following RT (Bentzen, 2006).

The ataxia-telangiectasia mutated $(A T M)$ gene encodes a protein kinase which plays a major function in the activation of cellular responses to DNA double-strand breaks by DNA repair or apoptosis (Prokopcova et al., 2007). Earlier studies reported that the ATM gene might be responsible for up to $8 \%$ of all breast cancer cases and the loss of heterozygosity often arises in the chromosomal section 11q22-23 in breast tumors (Meyn, 1999). 
Reports also showed that patients having a truncating mutation in both copies of the ATM gene may develop tissue toxicity if treated with RT (Mayrou et al., 2008).

The ataxia-telangiectasia mutated $(A T M)$ gene encodes a protein kinase which plays a major function in the activation of cellular responses to DNA double-strand breaks by DNA repair or apoptosis (McKinnon, 2004). Earlier studies reported that assessed the ATM gene might be responsible for up to $8 \%$ of all breast cancer cases and the loss of heterozygosity often arises in the chromosomal section 11q22-23 in breast tumors (Apostolou and Fostira, 2013). Reports also showed that patients having a truncating mutation in both copies of the ATM gene may develop tissue toxicity if treated with RT (Andreassen et al., 2016).

\subsection{Body weight}

Contributes to the process of wound healing, but also can lead to angiogenesis. This angiogenesis may lead to the growth of previously dormant metastatic cells (Baum et al., 2005). Normally, RT deteriorates the ability of cancer cells to create angiogenesis and, therefore, deteriorates the ability to metastasis. Starting RT too early after surgery may lead to the repair of this kind of radiation-induced damage and may halt the deterioration of angiogenesis.

\subsection{Age}

The mammary gland is highly sensitive to radiation-associated carcinogenesis, especially when exposures at young ages. The risk of contralateral breast cancer also finds higher in $<45$ years aged women after 10 years of receiving radiation (Fraass et al., 1985; Boice et al., 1992; Hankey et al., 1983). Besides this, in the case of early side effects, a higher prevalence of sleep problems and nausea is documented in younger patients after RT compared to the older patients (Hickok et al., 1996; Morrow, 1989).

Some previous evidence also indicated that older breast cancer patients treated with RT resulted in a lower occurrence of pain in general when compared with younger breast cancer individuals (Tesarova, 2013). Lundstedt et al. (2012) reported that young age is related to a greater possibility of breast pain up to 17 years after RT (Lundstedt et al., 2012). Gartner et al. (2009) from their study on 3,253 Danish breast cancer individuals found that radiotherapy at a young age was associated with a high risk of pain (Gartner et al., 2009). Peuckmann et al. also showed similar results in a study with 1,316 women. In this study, the breast cancer patients treated with RT resulted in chronic pain (duration $>6$ months) at different parts of the body. Besides, they accepted that RT and young age were particularly linked with a high risk of pain (Peuckmann et al., 2009). Furthermore, enough data indicate that age greatly influences whether a woman will result in lifelong RTinduced breast pain (Schroevers et al., 2006).

\subsection{Involvement of other diseases}

Inherited disorders such as ataxia-telangiectasia (AT) and Nijmegen breakage syndrome (NBS) are found to be associated with severe side effects of radiation, including an increased cancer risk after RT. In these disorders, the enhanced reaction to radiation could be demonstrated on the cellular level. For example, mutations in the ATM gene, encode a serine/threonine-protein kinase that is recruited and activated in response to DSBs and causes severe injury reactions in patients taking RT (Taylor et al., 1975). This indicates that rare cases of extreme sensitivity to radiation have a direct link with genetic disorders (Alsbeih et al., 2003; Andreassen et al., 2009).

\section{Translational approach}

From the above text, it is clear that some patients develop severe late RT induced side effects while others experience mild side effects only. It is also clear that genetic makeup helps to define a patient's radiosensitivity and is an important trigger for generating side effects. Therefore, the development of a prognostic tool to identify radiosensitive patients based on their genetic factors may allow personalized cancer treatment. Here a term is introduced called Radiogenomics, which is a genome-wide approach to characterize genetic predictors responsible for adverse radiotherapy effects. The goals of Radiogenomics are to 1) develop a predictive assay for identifying cancer patients who are most likely to develop severe radiotherapy side effects resulting from treatment with a standard RT protocol, and 2) to gather information on the molecular pathways which are responsible for radiation-induced tissue toxicities (West et al., 2010; West and Rosenstein, 2010). Radiogenomics studies are still in early stages; however identifying a large number of SNPs that have been replicated and validated in large, diverse cohorts may augment its chance to transit from bench to bedside (Ritchie, 2012). 


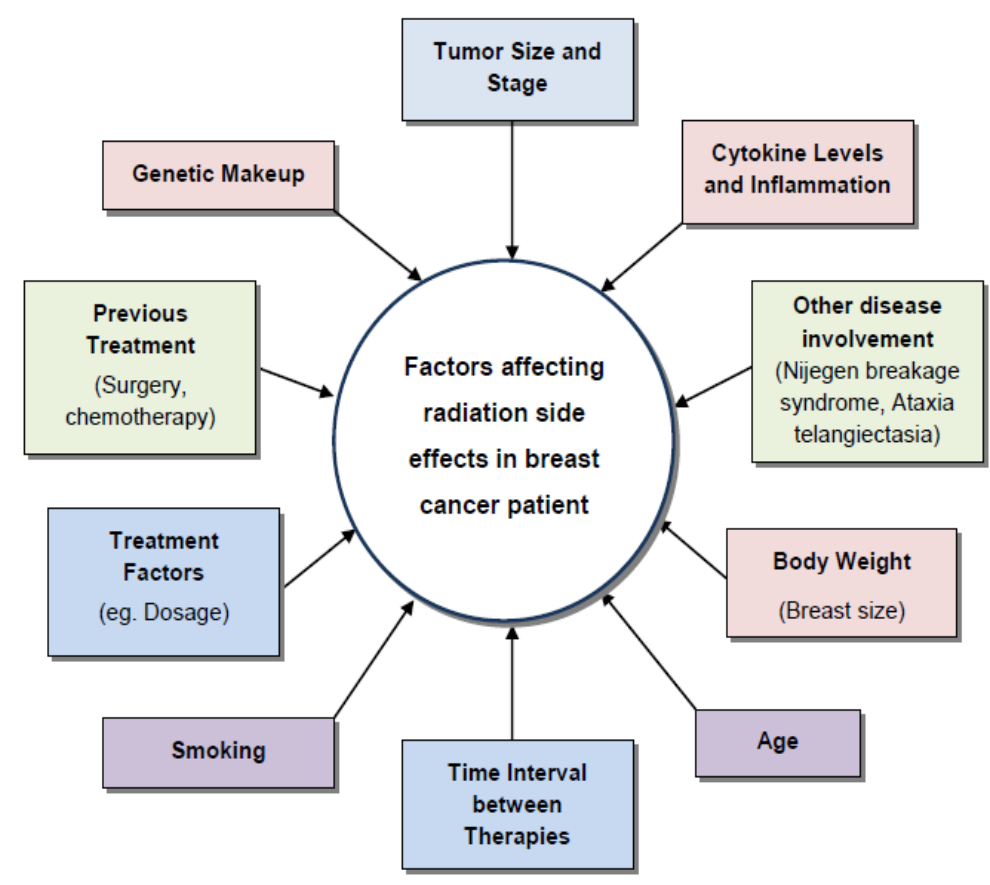

Figure 2. Factors affecting the side effects of radiation in breast cancer patients.

\section{Conclusions}

Breast cancer radiotherapy is associated with varying degrees of direct side effects in conjunction with significant indirect factors. During radiotherapy along with tumor cells normal tissues are also exposed to radiation. Damage of these normal tissues induces both local and systemic responses manifested by acute radiation toxicity. The risks of RT can be fully understood only after long-term follow-up studies. The factors affecting the risks of RT become increasingly important issues in the management of patients with early breast cancer. Factors associated with inflammation are characterized as major molecules for producing systemic responses to irradiation. Thus, the molecules which are directly or indirectly associated with inflammation could be used as a sensitive marker to detect exposure to radiation and monitor radiation-induced toxicity. A wide evaluation of SNPs, which is correlated with acute or late radiation sensitivity may also help to develop a useful tool for assessing breast cancer risk and also for predicting the complications related to conventional radiotherapy. In conclusion, it can be said that radiation therapies while helping in dramatic improvements in breast cancer-specific mortality, also increases the risk of side effects. It is therefore important to have a good knowledge of the radiotherapy-induced side effects and their associated factors to limit them.

\section{Conflict of interest}

None to declare.

\section{References}

Adams MJ, PH Hardenbergh, LS Constine and SE Lipshultz, 2003. Radiation-associated cardiovascular disease. Crit. Rev. Oncol. Hematol., 45: 55-75.

Ahsan H and AI Neugut, 1998. Radiation therapy for breast cancer and increased risk for esophageal carcinoma. Ann. Intern. Med., 128: 114-117.

Alsbeih G, MD Story, MH Maor, FB Geara and WA Brock, 2003. Chromosomal fragility syndrome and family history of radio-sensitivity as indicators for radiotherapy dose modification. Radiother. Oncol., 66: 341-344.

Alsner J, CN Andreassen and J Overgaard, 2008. Genetic markers for prediction of normal tissue toxicity after radiotherapy. Semin. Radiat. Oncol., 18: 126-135.

Andreassen, N Christian, SR Barry, LK Sarah, O Harry, DR Dirk, AC Jamie and CB Gillian, 2016. Individual patient data meta-analysis shows a significant association between the ATM rs1801516 SNP and toxicity after radiotherapy in 5456 breast and prostate cancer patients. Radiat. Oncol., 121: 431-439.

Andreassen CN and J Alsner, 2009. Genetic variants and normal tissue toxicity after radiotherapy: A systematic review. Radiother. Oncol., 92: 299-309. 
Anglian Breast Cancer Study Group, 2000. Prevalence and penetrance of BRCA1 and BRCA2 mutations in a population-based series of breast cancer cases. Br. J. Cancer., 83: 1301.

Antoniou A, PD Pharoah, S Narod, HA Risch, JE Eyfjord, JL Hopper and B Pasini, 2003. Average risks of breast and ovarian cancer associated with BRCA1 or BRCA2 mutations detected in case series unselected for family history: a combined analysis of 22 studies. Am. J. Hum. Genet., 72: 1117-1130.

Aparicio T, R Baer and J Gautier, 2014. DNA double-strand breaks repair pathway choice and cancer. DNA Repair, 19: 169-175.

Apostolou P, and F Fostira, 2013. Hereditary breast cancer: the era of new susceptibility genes. Biomed Res. Int., 747318.

Bandyopadhyay U, D Das and RK Banerjee, 1999. Reactive oxygen species: oxidative damage and pathogenesis. Curr. Sci., 77: 658-666.

Baskar R, KA Lee, R Yeo and KW Yeoh, 2012. Cancer and radiation therapy: current advances and future directions. Int. J. Med. Sci. Public Health, 9: 193-199.

Bassing $\mathrm{CH}$ and FW Alt, 2004. The cellular response to general and programmed DNA double strand breaks. DNA Repair, 3: 781-796.

Batar B, G Guven, S Eroz, NS Bese and M Guven, 2016. Decreased DNA repair gene XRCC1 expression is associated with radiotherapy-induced acute side effects in breast cancer patients. Gene, 582: 33-37.

Baum M, R Demicheli, W Hrushesky and M Retsky, 2005. Does surgery unfavourably perturb the 'natural history' of early breast cancer by accelerating the appearance of distant metastases? Eur. J. Cancer., 41: 508515.

Bentzen SM, 2006. Preventing or reducing late side effects of radiation therapy: radiobiology meets molecular pathology. Nat. Rev. Cancer, 6: 702-713.

Beral V, D Bull, R Doll, R Peto, G Reeves, PA Brandt and RA Goldbohm, 2004. Collaborative Group on Hormonal Factors in Breast cancer: Breast cancer and abortion: collaborative reanalysis of data from 53 epidemiological studies, including 83000 women with breast cancer from 16 countries. Lancet, 363: 10071016.

Boice JD, EB Harvey and M Blettner, 1992. Cancer in the contralateral breast after radiotherapy for breast cancer. N. Engl. J. Med., 326: 781-785.

Borrego SG, R Ortiz-López and A Rojas-Martínez, 2015. Ionizing radiation-induced DNA injury and damage detection in patients with breast cancer. Genet. Mol. Biol., 38: 420-432.

Bovelli D, G Plataniotis, FE Roila and ESMO Guidelines Working Group, 2010. Cardiotoxicity of chemotherapeutic agents and radiotherapy-related heart disease: ESMO Clinical Practice Guidelines. Ann. Oncol., 21: 277-282.

Brock WA and SL Tucker, 2000. In vitro radiosensitivity and normal tissue damage. Radiother. Oncol., 55: 9394.

Brown L, 1983. National radiation survey in the UK: indoor occupancy factors. Radiat. Prot. Dosim., 5: 203208.

Candeias SM and I Testard, 2015. The many interactions between the innate immune system and the response to radiation. Cancer Lett., 368: 173-178.

Chen Y, J Williams, I Ding, E Hernady, W Liu, T Smudzin, JN Finkelstein, P Rubin and P Okunieff, 2002. Radiation pneumonitis and early circulatory cytokine markers. Semin. Radiat. Oncol., 12: 26-33.

Di-Maggio FM, L Minafra, GI Forte, FP Cammarata, D Lio, C Messa, MC Gilardi and V Bravata, 2015. Portrait of inflammatory response to ionizing radiation treatment. J. Inflamm. Lond., 12: 14.

Donnelly EH, JB Nemhauser, JM Smith, ZN Kazzi, EB Farfán, AS Chang and SF Naeem, 2010. Acute radiation syndrome: assessment and management. South. Med. J., 103: 541-546.

Doody MM, JE Lonstein, M Stovall, DG Hacker, N Luckyanov, CE Land and US Scoliosis Cohort Study Collaborators, 2000. Breast cancer mortality after diagnostic radiography: findings from the US Scoliosis Cohort Study. Spine J., 25: 2052-2063.

Dunne-Daly CF, 1999. Principles of radiotherapy. Br. J. Hosp. Med. Lond., 74: 166-169.

Finnon P, S Kabacik, A MacKay, C Raffy, R Hern, R Owen and S Bouffler, 2012. Correlation of in vitro lymphocyte radiosensitivity and gene expression with late normal tissue reactions following curative radiotherapy for breast cancer. Radiother. Oncol., 105: 329-336.

Fraass BA, PL Roberson and AS Lichter, 1985. Dose to the contralateral breast due to primary breast irradiation. Int. J. Radiat. Oncol. Biol. Phys., 11: 485-497.

Garcia M, SL Mulvagh, CN Merz, JE Buring and JE Manson, 2016. Cardiovascular disease in women: clinical perspectives. Circ. Res., 11: 1273-1293. 
Gärtner R, J Maj-Britt, N Jeanette, E Marianne, K Niels and K Henrik, 2009. Prevalence of and factors associated with persistent pain following breast cancer surgery. Jama., 302: 1985-1992.

Gordon KJ and GC Blobe, 1782. Role of transforming growth factor- $\beta$ superfamily signaling pathways in human disease. Biochim. Biophys. Acta., 4: 197-228.

Guo GS, FM Zhang, RJ Gao, R Delsite, ZH Feng and SN Powell, 2011. DNA repair and synthetic lethality. Int. J. Oral. Sci., 3: 176-179.

Hankey BF, RE Curtis and MD Naughton, 1983. A retrospective cohort analysis of second breast cancer risk for primary breast cancer patients with an assessment of the effect of radiation therapy. J. Natl. Cancer Inst., 70: 797-804.

Halazonetis TD, VG Gorgoulis and J Bartek, 2008. An oncogene-induced DNA damage model for cancer development. Science, 319: 1352-1355.

Harvey EB and LA Brinton, 1985. Second cancer following cancer of the breast in Connecticut, 1935-82. J. Natl. Cancer Inst., 68: 99-112.

Hershman DL, X Wang, R McBride, JS Jacobson, VR Grann and AI Neugut, 2006. Delay in initiating adjuvant radiotherapy following breast conservation surgery and its impact on survival. Int. J. Radiat. Oncol. Biol. Phys., 65: 1353-1360.

Hickok JT, GR Morrow, JA Roscoe, K Mustian and P Okunieff, 2005. Occurrence, severity, and longitudinal course of twelve common symptoms in 1129 consecutive patients during radiotherapy for cancer. J. Pain Symptom Manag., 30: 433-442.

Hickok JT, GR Morrow, S McDonald and AJ Bellg AJ, 1996. Frequency and correlates of fatigue in lung cancer patients receiving radiation therapy: implications for management. J. Pain Symptom Manag., 11: 370-377.

Hill MA, 2004. The variation in biological effectiveness of X-rays and gamma rays with energy. Radiat. Prot. Dosim., 11: 471-481.

Hooning MJ, A Botma and BM Aleman, 2007. Long-term risk of cardiovascular disease in 10-year survivors of breast cancer. J. Natl. Cancer Inst., 99: 365-375.

Johansson S, H Svensson and J Denekamp, 2000. Timescale of evolution of late radiation injury after postoperative radiotherapy of breast cancer patients. Int. J. Radiat. Oncol. Biol. Phys., 48: 745-750.

Knaul FM, G Nigenda, R Lozano, H Arreola-Ornelas, A Langer and J Frenk, 2009. Breast cancer in Mexico: a pressing priority. Public Health, 51: 335-344.

Land CE, M Tokunaga, K Koyama, M Soda, DL Preston, I Nishimori and S Tokuoka, 2003. Incidence of female breast cancer among atomic bomb survivors, Hiroshima and Nagasaki, 1950-1990. Radiat. Res., 160: 707-717.

Lin C, SS Donaldson, JL Meza, JR Anderson, ER Lyden, CK Brown and K Morano, 2012. Effect of radiotherapy techniques (IMRT vs. 3D-CRT) on outcome in patients with intermediate-risk rhabdomyosarcoma enrolled in COG D9803-a report from the Children's Oncology Group. Int. J. Radiat. Oncol. Biol. Phys., 82: 1764-1770.

Lipshultz SE, MJ Adams, SD Colan, LS Constine, EH Herman, DT Hsu and KC Oeffinger, 2013. Long-term cardiovascular toxicity in children, adolescents, and young adults who receive cancer therapy: pathophysiology, course, monitoring, management, prevention, and research directions: a scientific statement from the American Heart Association. Circulation, 128: 1927-1995.

Little JB, 1998. Radiation-induced genomic instability. Int. J. Radiat. Biol., 74: 663-671.

Lliakis G, 1991. The role of DNA double strand breaks in lonizing radiation-induced killing of eukaryotic cells. Bioessays, 13: 641-648.

Lundstedt D, M Gustafsson, G Steineck, P Malmström, D Alsadius, A Sundberg and P Karlsson, 2012. Risk factors of developing long-lasting breast pain after breast cancer radiotherapy. Int. J. Radiat. Oncol. Biol. Phys., 83: 71-78.

Marks LB, X Yu and RG Prosnitz, 2005. The incidence and functional consequences of RT-associated cardiac perfusion defects. Int. J. Radiat. Oncol. Biol. Phys., 63: 214 -223.

Marks LB, D Hollis and M Munley, 2000. The role of lung perfusion imaging in predicting the direction of radiation-induced changes in pulmonary function tests. Cancer, 88: 2135-2141.

Mathes SJ and J Alexander, 1996. Radiation injury. Surg. Oncol. Clin. N. Am., 5: 809-824.

Mavrou A, GT Tsangaris, E Roma and A Kolialexi, 2008. The ATM gene and ataxia telangiectasia. Anticancer Res., 28: 401-405.

Mazurik VK and VF Mikhailov, 2001. Radiation-induced genome instability: phenomenon, molecular mechanisms, pathogenic significance. Radiat. Biol. Radioecol., 41: 272-289.

McKinnon PJ. ATM and ataxia telangiectasia. EMBO Reports, 5: 772-776. 
Meyn MS, 1999. Ataxia-telangiectasia, cancer and the pathobiology of the ATM gene. Clin. Genet., 55: 289304.

Mladenov E, S Magin, A Soni and G Iliakis, 2013. DNA double-strand break repair as determinant of cellular radiosensitivity to killing and target in radiation therapy. Front. Oncol., 3: 113.

Mohan G, A Hamna, AJ Jijo, S Devi, A Narayanasamy and B Vellingiri, 2019. Recent advances in radiotherapy and its associated side effects in cancer-a review. J. Basic Appl. Zool., 80: 1-10.

Motwani SB, EA Strom and NR Schechter, 2006. The impact of immediate breast reconstruction on the technical delivery of postmastectomy radiotherapy. Int. J. Radiat. Oncol. Biol. Phys., 66: 76-82.

Neugut AI, MD Weinberg, H Ahsan and J Rescigno, 1999. Carcinogenic effects of radiotherapy for breast cancer. Oncology, 13: 1245-1256.

Newman B, H Mu, LM Butler, RC Millikan, PG Moorman and M King, 1998. Frequency of breast cancer attributable to BRCA1 in a population-based series of American women. Jama., 279: 915-921.

Peuckmann V, E Ola, S Per, KR Niels, C Peer, M Susanne and G Mogens, 2009. Health care utilisation and characteristics of long-term breast cancer survivors: nationwide survey in Denmark. Eur. J. Cancer., 45: 625633.

Pierce LJ and BG Haffty, 2011. Radiotherapy in the treatment of hereditary breast cancer. Semin. Radiat. Oncol., 21: 43-50.

Popanda O, JU Marquardt, J Chang-Claude and P Schmezer, 2009. Genetic variation in normal tissue toxicity induced by ionizing radiation. Mutat. Res., 667: 58-69.

Powell SN, H Willers and F Xia, 2002. BCRA2 keeps Rad51 in line: high fidelity homologous recombination prevents breast and ovarian cancer? Mol. Cell., 10: 1262-1263.

Preston DL, Y Shimizu, DA Pierce, A Suyama and K Mabuchi, 2003. Studies of mortality of atomic bomb survivors. Report 13: Solid cancer and noncancer disease mortality: 1950-1997. Radiat. Res., 160: 381-407.

Price JG, J Idoyaga, H Salmon, B Hogstad, CL Bigarella, S Ghaffari, M Leboeuf and M Merad, 2015. CDKN1A regulates Langerhans cell survival and promotes $\mathrm{T}_{\text {reg }}$ cell generation upon exposure to ionizing irradiation. Nat. Immunol., 16: 1060-1071.

Prokopcova J, Z Kleibl, CM Banwell and P Pohlreich, 2007. The role of ATM in breast cancer development. Breast Cancer Res. Treat., 104: 121-128.

Redon CE, AJ Nakamura, YW Zhang, JJ Ji, WM Bonner, RJ Kinders, RE Parchment, JH Doroshow and Y Pommier, 2010. Histone $\gamma \mathrm{H} 2 \mathrm{AX}$ and poly (ADP-ribose) as clinical pharmacodynamic biomarkers. Clin. Cancer Res., 16: 4532-4542.

Ritchie MD, 2012. The success of pharmacogenomics in moving genetic association studies from bench to bedside: study design and implementation of precision medicine in the post-GWAS era. Hum. Genet., 131: 1615-1626.

Rojo F, J Garcia-Parra, S Zazo, I Tusquets, J Ferrer-Lozano, S Menendez and F Lobo, 2012. Nuclear PARP-1 protein overexpression is associated with poor overall survival in early breast cancer. Ann. Oncol., 23: 11561164.

Rosen EM, S Fan and S Rockwell, 1999. The molecular and cellular basis of radiosensitivity: Implications for understanding how normal tissues and tumors respond to therapeutic radiation. Cancer Invest., 17: 56-72.

Rosenstein BS, 2011. Identification of SNPs associated with susceptibility for development of adverse reactions to radiotherapy. Pharmacogenomics J., 12: 267-275.

Roychoudhuri R, H Evans, D Robinson and H Møller, 2004. Radiation-induced malignancies following radiotherapy for breast cancer. Br. J. Cancer, 91: 868-872.

Sasco AJ, R Kaaks and RE Little, 2003. Breast cancer: occurrence, risk factors and hormone metabolism. Expert Rev. Anticancer Ther., 4: 546-562.

Suzuki K, M Ojima, S Kodama and M Watanabe, 2003. Radiation-induced DNA damage and delayed induced genomic instability. Oncogene, 22: 6988-6993.

Schaue D, and WH McBride, 2015. Opportunities and challenges of radiotherapy for treating cancer. Nat. Rev. Clin. Oncol., 12: 527-540.

Schaue D, EL Kachikwu and WH McBride, 2012. Cytokines in radiobiological responses: a review. J. Radiat. Res., 178: 505-523.

Schroevers M, AV Ranchor and R Sanderman, 2006. Adjustment to cancer in the 8 years following diagnosis: a longitudinal study comparing cancer survivors with healthy individuals. Soc. Sci. Med., 63: 598-610.

Smith KL and C Isaacs, 2007. Management of women at increased risk for hereditary breast cancer., Breast. Dis., 27: 51-67. 
Taylor AM, DG Harnden, CF Arlett, SA Harcourt, AR Lehmann, S Stevens and BA Bridges, 1975. Ataxia telangiectasia: a human mutation with abnormal radiation sensitivity. Nature, 258: 427-429.

Tesarova P, 2013. Breast cancer in the elderly-Should it be treated differently? Rep. Pract. Oncol. Radiother., 18: 26-33.

Turesson I, J Nyman, E Holmberg and A Odén, 1996. Prognostic factors for acute and late skin reactions in radiotherapy patients. Int. J. Radia.t Oncol. Biol. Phys., 36: 1065-1075.

Wang J, M Boerma, Q Fu and M Hauer-Jensen, 2007. Significance of endothelial dysfunction in the pathogenesis of early and delayed radiation enteropathy. World J. Gastroenterol., 13: 3047-3055.

Weintraub NL, WK Jones and D Manka, 2010. Understanding Radiation-Induced Vascular Disease. J. Am. Coll. Cardiol., 55: 1237-1239.

West C and BS Rosenstein, 2010. Establishment of a radiogenomics consortium. Radiother. Oncol., 94: 117118.

West C, BS Rosenstein, J Alsner, D Azria, G Barnett and A Begg, 2010. Establishment of a Radiogenomics Consortium. Int. J. Radiat. Oncol. Biol. Phys., 76: 1295-1296.

West CM and GC Barnett, 2011. Genetics and genomics of radiotherapy toxicity: towards prediction. Genome Med., 3: 52.

Wygoda A, B Maciejewski, K Skladowski, M Hutnik, B Pilecki, M Golen and T Rutkowski, 2009. Pattern analysis of acute mucosal reactions in patients with head and neck cancer treated with conventional and accelerated irradiation. Int. J. Radiat. Oncol. Biol. Phys., 73: 384-390.

Yang Y, Alexandr VB, J Werner and S Karakhanova, 2013. Reactive oxygen species in the immune system. Immunol. Rev., 32: 249-270.

Youlden DR, SM Cramb, NAM Dunn, JM Muller, CM Pyke and PD Baade, 2012. The descriptive epidemiology of female breast cancer: an international comparison of screening, incidence, survival and mortality. Cancer Epidemiol., 36: 237-248.

Youlden DR, SM Cramb, CH Yip and PD Baade, 2014. Incidence and mortality of female breast cancer in the Asia-Pacific region. Cancer Biol. Med., 11: 101-115.

Yu H, 2012. Typical Cell Signaling Response to Ionizing Radiation: DNA Damage and Extranuclear Damage. Chin. J. Cancer, 24: 83-89.

Zablotska LB, C Amitabh, D Ananya and IN Alfred, 2005. Increased risk of squamous cell esophageal cancer after adjuvant radiation therapy for primary breast cancer. Am. J. Epidemiol., 161: 330-337.

Zagar TM, DM Cardinale and LB Marks, 2016. Breast cancer therapy-associated cardiovascular disease. Nat. Rev. Clin. Oncol., 13: 172-184. 